\title{
Local anaesthetic effect of topical amethocaine gel in neonates: randomised controlled trial
}

Anoo Jain, Nicholas Rutter

\begin{abstract}
Aim-To assess the efficacy of amethocaine as a topical local anaesthetic in neonates.

Methods-A randomised, double blind controlled trial compared $4 \%$ amethocaine gel (Ametop) with placebo in 60 healthy neonates ( 29 to 42 weeks of gestation) in the first week after birth. Either $1.5 \mathrm{~g} \mathrm{4 \%} \mathrm{w} / \mathrm{w}$ amethocaine (gel) or $1.5 \mathrm{~g}$ placebo gel were applied to the dorsum of one foot. No gel was applied to the other foot. Each foot was occluded and left for one hour. Local anaesthesia was then assessed by eliciting the cutaneous withdrawal reflex in response to stimulation with a series of graded nylon filaments (von Frey hairs). The reflex was first elicited from the control and then the treated foot. The difference in filament thickness and deforming weight required to elicit the reflex was recorded.

Results-In infants treated with amethocaine, 17 of $31(54.8 \%)$ showed evidence of local anaesthetic action compared with five of $29(17.2 \%)$ in the placebo group $(p=0.003)$. The mean difference in deforming weight required to elicit the reflex was $18.8 \mathrm{~g}$ in the amethocaine group compared with $3.9 \mathrm{~g}$ in the placebo group $(p=0.02)$. The apparent local anaesthetic action of the placebo can be explained by habituation to repeated stimulation. Conclusions-It is concluded that topical amethocaine gel has a local anaesthetic action on neonatal skin which merits further investigation. An effective and safe surface local anaesthetic would be valuable for the relief of procedure related pain in neonates.

(Arch Dis Child Fetal Neonatal Ed 2000;82:F42-F45)
\end{abstract}

Keywords: amethocaine gel; pain; anaesthesia

Painful and invasive procedures are commonly performed in neonates, especially in intensive care units. Heel prick blood sampling and venepuncture are the most common procedures that pierce the skin; they are needed for the routine care of healthy term and sick premature infants alike. ${ }^{1}$ Modification of the technique of sampling plays some part in pain reduction, ${ }^{2}$ but a topical local anaesthetic would be very useful. Topical local anaesthetics are widely used to relieve the pain of venepuncture in children, but their use in neonates has been less successful. A successful topical local anaesthetic should be quick acting, effective, safe and should not interfere with the proce- dure involved. Studies on lignocaine and lignocaine/prilocaine eutectic mixture in neonates have produced disappointing results. ${ }^{3-8}$ Amethocaine is an alternative. It can provide effective and safe surface local anaesthesia in children. ${ }^{9}$ There is no information on the effectiveness of amethocaine in neonates, so we have explored its efficacy in this age group.

\section{Methods}

We conducted a randomised double blind controlled trial. Surface local anaesthesia was assessed by eliciting the cutaneous withdrawal reflex in response to stimulation of the dorsum of the foot. To provide a graded range of stimuli, we used a series of 20 von Frey hairs. The von Frey hair consists of a flexible nylon filament attached at right angles to one end of a perspex holder. Each filament has a different thickness, ranging from very fine to thick. The nylon filament is placed on to the dorsum of the foot and deformed by downward pressure. A measurable and reproducible weight is required to deform the filament. When the stimulus exceeds the threshold of the reflex, the cutaneous withdrawal reflex is elicited. This reflex consists of fanning of the toes, followed by flexion of the ankle, knee and hip and withdrawal of the leg.

Our protocol is summarised in fig 1. For every subject, the baby's right foot acted as the untreated control and the left as the treated foot. We applied a marker ring to the dorsum of each foot. This defined and exposed a circular area of skin $1 \mathrm{~cm}$ in diameter which was used for testing. On the treated foot within the marker ring, we applied either $1.5 \mathrm{~g}$ of placebo or $1.5 \mathrm{~g}$ of $4 \%$ amethocaine gel (Ametop). The placebo was identical in every way except for the absence of amethocaine. We covered each foot with an occlusive dressing and left the baby alone (fig 1A). An hour later, the occlusive dressings were removed and any excess gel wiped from the treated foot. The control foot was always tested first. The grade of filament was increased until the cutaneous withdrawal reflex was elicited (fig 1B). The filament number and its corresponding deforming weight were recorded. This was repeated in the same way on the treated foot until the reflex was again elicited (fig 1C). The difference in thickness of filament required to elicit the cutaneous withdrawal reflex from the treated and the control foot was used to determine the presence of a local anaesthetic action. The difference in corresponding deforming weights was used as a measure of the strength of this action.

We defined local anaesthetic action by studying a reference group of 30 infants in 
(A)
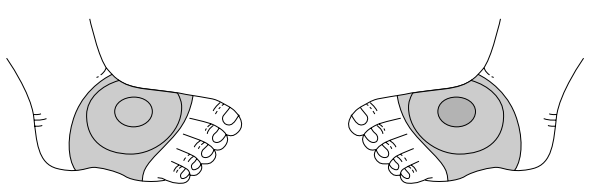

(B)
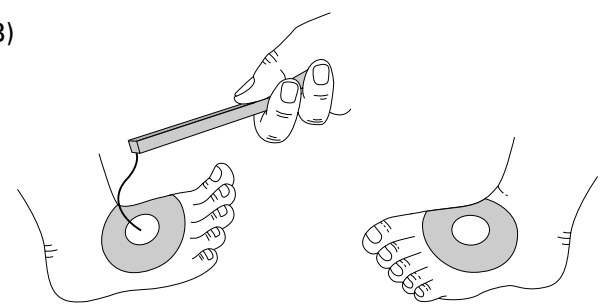

(C)
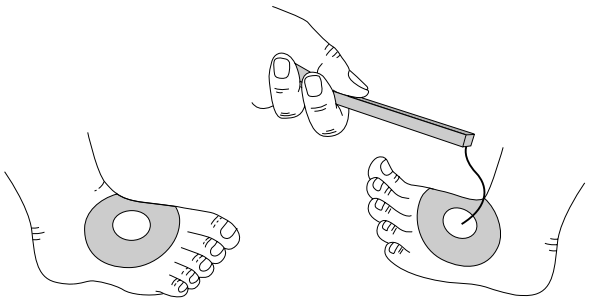

Figure 1 Protocol used for this study: in each pair the right foot is the control foot and the left, the treated foot. (A) Application of gel to the treated foot; (B) eliciting the cutaneous withdrawal reflex from the control foot; $(C)$ eliciting the cutaneous withdrawal reflex from the treated foot.

Table 1 Difference in von Frey hair number required to elicit reflex from control and treated foot in amethocaine, placebo, and reference groups

\begin{tabular}{lcrrrrrrrr}
\hline Hair difference & -1 & \multicolumn{1}{l}{0} & 1 & 2 & 3 & 4 & 5 & 6 & 7 \\
\hline Amethocaine $(\mathrm{n}=31)$ & 1 & 6 & 7 & 5 & 6 & 3 & 1 & 1 & 1 \\
Placebo $(\mathrm{n}=29)$ & 0 & 12 & 12 & 2 & 1 & 2 & & & \\
Reference (n=30) & 1 & 17 & 11 & 1 & & & & & \\
\hline
\end{tabular}

whom neither placebo nor amethocaine gel was applied to either foot. Using the same protocol, habituation to repeated stimulation was demonstrated, with an increase in threshold between the right and left foot of one filament thickness in $11 / 30(36 \%)$ and two filament thickness in $1 / 30(3.3 \%)$. Local anaesthetic action was therefore defined as the need for a filament thickness of at least two more grades to elicit the reflex from the treated foot.

We studied 60 healthy infants between May and August 1998. We stratified the subjects by gestation into three groups of 20: term (below 37 weeks of gestation), mildly preterm (33 to 37 weeks of gestation), and moderately preterm (28 to 32 weeks of gestation). They ranged from 29 to 42 weeks of gestation and weighed from 1.03 to $4.62 \mathrm{~kg}$. They were studied in the first week after birth by the same observer. Infants who were admitted to the postnatal wards or neonatal intensive care at Nottingham City Hospital were eligible for entry, but we excluded infants who were unwell, ventilated, or sedated. Infants in the reference group, in whom local anaesthetic action was defined, ranged from 31 to 42 weeks of gestation and weighed from 1.49 to $4.5 \mathrm{~kg}$.

The study was approved by the local ethics committee. Informed and written parental consent was obtained. In addition we obtained exemption from the restrictions of the product licence from the Medicines Control Agency.

In each group half the infants were randomly allocated to have amethocaine applied to the dorsum of the foot and the other half to placebo. Placebo and amethocaine were packaged into identical tubes by the hospital pharmacy who randomised and coded them. The code was only broken at the end of the study.

Statistical analysis was performed using SPSS 8.0 software. Comparison of the median von Frey hair numbers in each group and the proportions within the amethocaine and placebo groups who showed a local anaesthetic action was made using Mann-Whitney $U$ and $\chi^{2}$ tests, respectively. The difference in mean deforming weight required to elicit the reflex from the placebo and amethocaine groups was analysed using the unpaired Student's $t$ test.

\section{Results}

The actual differences in von Frey hair number required to elicit the cutaneous withdrawal reflex from control and treated feet for the amethocaine, placebo, and reference groups are shown in table 1 . The median difference was one hair for the placebo group and none for the reference group. For the amethocaine group it was two. This difference was significant $(p=0.005)$. When local anaesthetic action was defined as a difference in filament thickness of two or more, 17 of 31 (54.8\%) in the amethocaine group showed local anaesthetic action compared with five of $29(17.2 \%)$ in the placebo group $(p=0.003)$. We compared the strength of local anaesthetic action in the two groups by recording the difference in deforming weight required to elicit the reflex from the control or treated foot (fig 2). In the placebo group the mean weight difference required to elicit the cutaneous withdrawal reflex was $3.9 \mathrm{~g}$. In the amethocaine group it was $18.9 \mathrm{~g}$. This fivefold difference was significant $(p=0.02)$.

Two infants in the amethocaine group (one of 34 weeks of gestation, one of 32 weeks of gestation, weighing $2.53 \mathrm{~kg}$ and $1.75 \mathrm{~kg}$, respectively) had minor and transient reactions. They developed localised erythema at the site of amethocaine application. Both infants remained well and the erythema disappeared 20 minutes after the amethocaine had been removed.

\section{Discussion}

Invasive procedures that pierce the skin of neonates are very common. Capillary heel prick blood sampling is the most frequently performed painful procedure in the newborn intensive care unit ${ }^{1}$ and the postnatal ward. It is probably more painful than venepuncture, the next most frequently performed procedure. ${ }^{10} 11$ The use of a lignocaine/prilocaine cream (EMLA) to relieve the pain from such procedures has been very successful in children, particularly with venepuncture. However, its use in neonates has been less successful, with no or little effect in heel pricks ${ }^{45}$ and no or limited effect in venepuncture. ${ }^{6-8}$ There 
are also concerns about its safety because the prilocaine component may cause methaemoglobinaemia, ${ }^{12-15}$ although the dangers may be theoretical rather than real in the context of topical skin use. ${ }^{6}{ }^{12}$ Amethocaine as a $4 \% \mathrm{w} / \mathrm{w}$ gel (Ametop) has been developed for use as a topical local anaesthetic by McCafferty. ${ }^{16}$ It is licensed for use in term infants over one month of age. Structurally it has a lipophilic benzene ring attached to a tertiary amine group by an ester link chain. This differs from local anaesthetics like lignocaine and prilocaine which have an amide link chain. It acts by blocking the influx of sodium ions across the axon and thus the propagation of the action potential along the nerve. It is rapidly metabolised in the blood by plasma pseudo-cholinesterase. In vivo studies in adults have shown that the pain response to insertion of a needle is reduced by topical amethocaine. ${ }^{16}$ In a large study in children application of topical amethocaine gel produced effective local anaesthesia for venepuncture in $89 \% .^{9}$ Transient localised erythema occurred in $6.3 \%$ and a localised urticarial reaction in $0.6 \%$. The plasma concentration of amethocaine and its metabolites has been studied and was unrecordable in seven of 10 subjects. In those in whom amethocaine was detected no significant side effects were reported. ${ }^{17}$ Since then more than 130000 applications of amethocaine have been used in over 25000 children with no serious adverse effects. ${ }^{18}$ There are few data about the use of amethocaine in neonates. In a pilot study of 20 healthy preterm infants using $1 \%$ amethocaine ointment, Barker and Rutter found some evidence of percutaneous absorption with an increase in local skin blood flow, but local anaesthetic action was not assessed. ${ }^{19}$ This vasodilator action of amethocaine might be a practical advantage over the vasoconstrictor effect of lignocaine and prilocaine during blood sampling.

The cutaneous withdrawal reflex is a convenient objective way of testing local anaesthetic action in neonates. ${ }^{30-22}$ It is not known whether touch or pain fibres are stimulated, but this is of no particular relevance in a local anaesthetic study. Habituation to the stimulus is well described and explains the apparent local anaesthetic action of the placebo gel in our study. The reference group showed similar habituation. In future studies which use this method of assessing local anaesthesia, it would be wise to compare placebo and active gel in each infant, randomising the order of testing. We chose to use an untreated foot because we were uncertain whether placebo gel itself might modify the cutaneous flexor reflex.

We have shown that the group treated with amethocaine had a local anaesthetic action in significantly more cases than the placebo group. Furthermore, the strength of this action was increased almost fivefold. Although amethocaine is effective after application for one hour, there is evidence that an application time of 30 minutes can provide satisfactory anaesthesia. ${ }^{916}$ We chose to study the gel one hour after application so we could be sure of its absorption through the skin, but there is no theoretical reason why the onset of action in neonates should differ from that of a child.

Two of our infants showed a transient localised erythema after application of the amethocaine gel. In each case, the reaction had disappeared 20 minutes later. Similar reactions have been reported in other trials of amethocaine. $^{92324}$ No systemic side effects were recorded in these nor any other subject in the amethocaine group. The area of skin that is anaesthetised is limited to the site of application of amethocaine. To the best of our knowledge, these are the only such reactions to have been reported in neonates.

We conclude that topical amethocaine gel has a local anaesthetic action on neonatal skin. This is the first time that this action has been shown in neonates and it merits further investigation. In particular, information about the time of onset, duration of action, and clinical efficacy is required. An effective and safe surface local anaesthetic would be a valuable asset for the relief of procedure related pain in the newborn.

We acknowledge the help of Dr DF McCafferty who provided us with the placebo and Mrs S Pacey and S Charlesworth from the pharmacy department at Nottingham City Hospital.

1 Barker DP, Rutter N. Exposure to invasive procedures in neonatal intensive care unit admissions. Arch Dis Child Fetal Neonatal Ed 1995;72:F47-8.

2 Harpin VA, Rutter N. Making heel pricks less painful. Arch Dis Child 1983;58:226-8.

3 Barker DP, Rutter N. Lignocaine ointment and local anaesthesia in preterm infants. Arch Dis Child Fetal Neonatal Ed 1995; 72:F203-4.

4 Rushforth JA, Griffiths G, Thorpe H, Levene MI. Can topical lignocaine reduce behavioural response to heel prick? Arch Dis Child Fetal Neonatal Ed 1995;72:F49-51.

5 Larsson BA, Jylli L, Lagercrantz H, Olsson GL. Does a local anaesthetic cream (EMLA) alleviate pain from heellancing in neonates? Acta Anaesthesiologica Scandinavica 1995;39:1028-31.

6 Taddio A, Ohlsson A, Einarson TR, Stevens B, Koren G. A systematic review of lidocaine-prilocaine cream (EMLA) in the treatment of acute pain in neonates. Pediatrics 1998;101:E1.

7 Acharya AB, Bustani PC, Phillips JD, Taub NA, Beattie RM. Randomised controlled trial of eutectic mixture of local anaesthetics cream for venepuncture in healthy preterm infants. Arch Dis Child Fetal Neonatal Ed 1998;78:F138-42.

8 Larsson BA, Tannfeldt G, Lagercrantz H, Olsson GL. Alleviation of the pain of venepuncture in neonates. Acta Paeviation of the pain of venepuncture in
diatrica Scandinavica 1998;87:774-9.

9 Woolfson AD, McCafferty DF, Boston V. Clinical experiences with a novel percutaneous amethocaine preparation: prevention of pain due to venepuncture in children. $\mathrm{Br} \mathcal{F}$ Clin Pharmacol 1990;30:273-9.

10 Larsson BA, Tannfeldt G, Lagercrantz H, Olsson GL. Venipuncture is more effective and less painful than heel lancing for blood tests in neonates. Pediatrics 1998;101:882-6.

11 Shah VS, Taddio A, Bennett S, Speidel BD. Neonatal pain response to heel stick vs venepuncture for routine blood sampling. Arch Dis Child Fetal Neonatal Ed 1997;77:F1434.

12 Brisman M, Ljung BML, Otterbom I, Larsson LE, Andreasson SE. Methaemoglobin formation after the use of EMLA in term neonates. Acta Paediatrica Scandinavica 1998;87:1191-4.

13 Duncan PG, Kobrinsky N. Prilocaine-induced methemoglobinemia in a newborn infant. Anesthesiology 1983;59:75-6.

14 Kumar AR, Dunn N, Naqvi M. Methemoglobinemia associated with a prilocaine-lidocaine cream. Clin Pediatr 1997;36:239-40

15 Lloyd CJ. Chemically induced methaemoglobinaemia in a neonate. Br f Oral Maxillofacial Surg 1992;30:63-5.

$16 \mathrm{McCafferty} \mathrm{DF}$, Woolfson $\mathrm{AD}$, Boston V. In vivo assessment of percutaneous local anaesthetic preparations. $\mathrm{Br} f$ Anaesthes 1989;62:17-21.

17 Mazumdar B, Tomlinson AA, Faulder GC. Preliminary study to assay plasma amethocaine concentrations after study to assay plasma amethocaine concentrations after
topical application of a new local anaesthetic cream topical application of a new local anaesthetic cream
containing amethocaine. Br f Anaesthes 1991;67:432-6. 
18 McCafferty DF, Woolfson AD. Pharmacists 'magic' gel is launched. The Pharmaceutical fournal 1996;256:219.

19 Barker DP, Rutter NR Skin blood flow and topical local anaesthesia in preterm infants. Early Hum Dev anaesthesia in

20 Andrews K, Fitzgerald $M$. The cutaneous withdrawal reflex in human neonates: sensitizatio effects of contralateral stimulation. Pain 1994;56:95-101.

21 Fitzgerald M, Shaw A, MacIntosh N. Postnatal development of the cutaneous flexor reflex: comparative study of preterm infants and newborn rat pups. Dev Med Child Neurol 1988;30:520-6.
22 Woolf CJ, Swett JE. The cutaneous contribution to the hamstring flexor reflex in the rat: an electrophysiological and anatomical study. Brain Res 1984;303:299-312.

23 Doyle E, Freeman J, Im NT, Morton NS. An evaluation of a new self-adhesive patch preparation of amethocaine for topical anaesthesia prior to venous children. Anaesthesia 1993;48:1050-2.

24 Lawson RA, Smart NG, Gudgeon AC, Morton NS. Evaluation of an amethocaine gel preparation for percutaneous analgesia before venous cannulation in children. $\mathrm{Br} \mathcal{F}$ Anaesthes 1995;75:282-5. 